\title{
Scalar field theory in $3+1$ dimensions
}

\author{
Roman Koniuk and Rolf Tarrach* \\ Department of Physics, York University, Toronto, Ontario, Canada M3J 1P3
}

(Received 31 August 1984)

\begin{abstract}
We study the existence of a stable ground state for the most general renormalizable single scalar field theory in four dimensions within a variational approach. In its regularized version we find a theory with an energy density which is not bound from below but with a metastable local minimum such that when the cutoff is removed the theory is interacting, finite, and possesses a stable ground state. In fact, we find that this theory is not stable unless it is symmetric. This generalizes Stevenson's recent results on even $\phi^{4}$ theory.
\end{abstract}

\section{INTRODUCTION}

Quantum field theory is essentially a theory of the vacuum. The knowledge of how the vacuum reacts to whatever external source we choose is enough for determining all the Green's functions of the theory. It may happen, however, that for a given Lagrangian there does not exist a ground state. The theory is unbounded, and thus meaningless, even though perturbation theory may be perfectly well behaved. Of course, perturbation theory makes use of a different vacuum that exists by definition as it only requires the existence of the free field theory. Surprisingly, after half a century of quantum field theory there is no interacting theory known to exist, i.e., have a ground state, in four dimensions. Even worse, our simplest field theory, $\lambda \phi^{4}$ theory, is very likely trivial (for $\lambda_{B}>0$ ): it only exists if the renormalized coupling constant is zero.

To the best of our knowledge the first reference to triviality is due to Wilson. ${ }^{1}$ The first rigorous proof was given by Aizenman for even $\phi^{4}$ theory in more than four dimensions. Fröhlich has generalized this result to twocomponent $\lambda|\phi|^{4}$ theories and nonlinear $\sigma$ models in $d>4$ dimensions and has given (with his collaborators) strong evidence for triviality in $d=4$ dimensions. ${ }^{3}$ There is also increasing numerical evidence for triviality of $\lambda \phi^{4}$ theory in $d=4$ dimensions. ${ }^{4}$ All this work assumes that the bare coupling constant is positive, so that the functional integrals that define the quantum theory are convergent.

Very recently, Stevenson, using variational methods, has offered a new view of this problem. ${ }^{5}$ Imagine a theory that is not bound from below in its regularized version but that has a metastable state whose lifetime goes to infinity when the cutoff is removed. In other words, the barrier that separates the metastable state from the unbounded well becomes infinitely wide when the cutoff is removed. He calls this kind of quantum field theory "precarious." For this kind of theory the regularized version is somewhat misleading: it is neither bound from below nor does it have a stable ground state. "A precarious theory is one which is unstable for any finite ultraviolet cutoff but which becomes stable when the cutoff is removed."5

Variational methods are ideally suited for investigating the ground-state energy for all values of the parameters.
They were introduced into quantum field theory by Schiff ${ }^{6}$ who, in fact, found that the renormalized coupling constant of $\lambda \phi^{4}$ theory had to go to zero as the cutoff was removed, if one demanded a stable ground state. However, Schiff concluded at that time that the trial wave functionals were not good enough. Barnes and Ghandour ${ }^{7}$ extended the method to fermions and introduced an unambiguous renormalization procedure into the variational method borrowed from the effective-potential technique. ${ }^{8}$ The trial wave functionals they used were of Gaussian type (for scalar fields), and they observed that Gaussians select the same class of diagrams as the leading-order diagrams in the $1 / N$ expansion in an $\mathrm{O}(N) \lambda|\phi|^{4}$ theory. A combination of both techniques has been used by Bardeen and Moshe $^{9}$ in their very complete study of the $O(N)$ $\lambda|\phi|^{4}$ model.

Stevenson, with the use of this very same Gaussian variational approach, finds that an interacting renormalized $\lambda \phi^{4}$ theory requires a negative, but vanishingly small, bare coupling constant. ${ }^{5}$ Although the classical potential is unbounded from below this theory turns out to have a stable, albeit precarious, ground state.

In three dimensions nontrivial theories are known to exist, e.g., $\phi^{6}$ theory. ${ }^{1,10}$ In four dimensions, $\lambda \phi^{4}$ theory seems to be, when it exists, either trivial or if nontrivial then precarious. The aim of this work is to study the most general, renormalizable, single scalar field theory in four dimensions within the variational Gaussian approach. It is an extension of Stevenson's work ${ }^{5}$ which includes in the Lagrangian odd terms in the fields. Our results are a generalization of his and show the same qualitative features. The theory is, when interacting, precarious.

\section{THE SEARCH FOR THE GROUND STATE}

The Hamiltonian density of the most general, renormalizable, single scalar field theory is

$\mathscr{H}=\frac{1}{2} \dot{\phi}^{2}+\frac{1}{2}|\nabla \phi|^{2}+a_{B} \phi+\frac{1}{2} m_{B}^{2} \phi^{2}+g_{B} \phi^{3}+\lambda_{B} \phi^{4}$,

where $m_{B}{ }^{2}$ can be negative. The variational computation of the ground-state energy density within the Gaussian approximation is straightforward. One writes 
$\phi(x)=\phi_{0}+\int \frac{d^{3} k}{(2 \pi)^{3} 2 \omega_{k}\left(\Omega^{2}\right)}\left[a_{\Omega}(\mathbf{k}) e^{-i k \cdot x}+a_{\Omega}^{\dagger}(\mathbf{k}) e^{i k \cdot x}\right]$,

where

$$
\begin{aligned}
& \omega_{k}\left(\Omega^{2}\right) \equiv\left(\mathbf{k}^{2}+\Omega^{2}\right)^{1 / 2}, \\
& {\left[a_{\Omega}(\mathbf{k}), a_{\Omega}^{\dagger}\left(\mathbf{k}^{\prime}\right)\right]=(2 \pi)^{3} 2 \omega_{k}\left(\Omega^{2}\right) \delta\left(\mathbf{k}-\mathbf{k}^{\prime}\right),} \\
& a_{\Omega}(\mathbf{k})\left|0_{\Omega}\right\rangle=0, \\
& \left\langle 0_{\Omega} \mid 0_{\Omega}\right\rangle=1,
\end{aligned}
$$

and $\Omega$ is as yet an undetermined function of the constant background field $\phi_{0}$. Then an upper bound to the ground-state energy density will be given by the minimum of

$$
\left.\mathscr{V}_{G}\left(\phi_{0}, \Omega\left(\phi_{0}\right)\right) \equiv\left\langle 0_{\Omega}|\mathscr{H}| 0_{\Omega}\right\rangle\right|_{\Omega=\Omega\left(\phi_{0}\right)},
$$

when $\phi_{0}$ is varied in the whole parameter space $\Omega\left(\phi_{0}\right)$, having been fixed in advance by minimization. The notation in Eq. (4) reminds us of the fact that the energy density computed in this way is nothing but the Gaussian effective potential. ${ }^{11}$ Of course Eq. (4) has UV divergences. These are of two kinds. One group is absorbed by renormalization of all the coefficients of the potential of Eq. (1). The residual UV divergences correspond to the zeropoint energy, which we just subtract.

The computation of Eq. (4) leads (in the notation of Ref. 5) to

$$
\begin{aligned}
\left\langle 0_{\Omega}|\mathscr{H}| 0_{\Omega}\right\rangle= & I_{1}\left(\Omega^{2}\right)+\frac{1}{2}\left(m_{B}{ }^{2}-\Omega^{2}\right) I_{0}\left(\Omega^{2}\right)+a_{B} \phi_{0} \\
& +\frac{1}{2} m_{B}{ }^{2} \phi_{0}{ }^{2}+g_{B} \phi_{0}{ }^{3}+\lambda_{B} \phi_{0}^{4} \\
& +3 g_{B} \phi_{0} I_{0}\left(\Omega^{2}\right)+6 \lambda_{B} \phi_{0}{ }^{2} I_{0}\left(\Omega^{2}\right) \\
& +3 \lambda_{B} I_{0}{ }^{2}\left(\Omega^{2}\right)
\end{aligned}
$$

where

$$
I_{n}\left(\Omega^{2}\right)=\int \frac{d^{3} k}{(2 \pi)^{3} 2 \omega\left(\Omega^{2}\right)}\left[\omega_{k}^{2}\left(\Omega^{2}\right)\right]^{n} .
$$

Notice that $I_{n}\left(\Omega^{2}\right)$ is divergent for $n \geq-1$. We will regularize the theory with a symmetric cutoff $\Lambda$. The domain of validity of the regularized theory is

$$
0 \leq \Omega^{2}, \phi_{0}^{2} \ll \Lambda^{2} \text {. }
$$

We will renormalize the theory at $\phi_{0}=0$. The stationary condition

$$
\frac{\partial \mathscr{V}_{G}}{\partial \Omega_{0}{ }^{2}}\left(0, \Omega_{0}\right)=\frac{1}{4} I_{-1}\left(\Omega_{0}{ }^{2}\right)\left[\Omega_{0}{ }^{2}-m_{B}{ }^{2}-12 \lambda_{B} I_{0}\left(\Omega_{0}{ }^{2}\right)\right]=0
$$

leads to either

$$
\Omega_{0}{ }^{2}-m_{B}{ }^{2}-12 \lambda_{B} I_{0}\left(\Omega_{0}{ }^{2}\right)=0, \quad \Omega_{0}{ }^{2} \geq 0
$$

or if Eq. (9) does not have solutions,

$$
\Omega(0) \equiv \Omega_{0}=0 \text {. }
$$

We consider the first case, Eq. (9). We define and find our renormalized parameters to be

$$
\begin{aligned}
\left.a \equiv \frac{d \mathscr{V}}{d \phi_{0}}\right|_{\phi_{0}=0}=a_{B} & +3 g_{B} I_{0}\left(\Omega_{0}{ }^{2}\right) \\
\left.m^{2} \equiv \frac{d^{2} \mathscr{V}}{d \phi_{0}^{2}}\right|_{\phi_{0}=0}= & \Omega_{0}{ }^{2}-3 g_{B} \Omega_{0}{ }^{2 \prime} I_{-1}\left(\Omega_{0}{ }^{2}\right) \\
& +\frac{1}{4}\left(\Omega_{0}{ }^{2 \prime}\right)^{2} I_{-1}\left(\Omega_{0}{ }^{2}\right) \\
& \times\left[1+6 \lambda_{B} I_{-1}\left(\Omega_{0}{ }^{2}\right)\right],
\end{aligned}
$$

where

$$
\left(\Omega_{0}^{2 \prime}\right)=\frac{6 g_{B}}{1+6 \lambda_{B} I_{-1}\left(\Omega_{0}^{2}\right)} .
$$

This value is obtained by minimization of $\mathrm{m}^{2}$, which is equivalent to fixing $\Omega\left(\phi_{0}\right)$ around $\phi_{0}=0$ by minimizing $\mathscr{V}_{G}\left(\phi_{0}, \Omega\right)$. Substitution of Eq. (13) into Eq. (12) leads to

$$
m^{2}=\Omega_{0}^{2}-9 \frac{g_{B}{ }^{2} I_{-1}\left(\Omega_{0}^{2}\right)}{1+6 \lambda_{B} I_{-1}\left(\Omega_{0}^{2}\right)} .
$$

The renormalized cubic coupling constant is given by

$$
\begin{aligned}
g=\left.\frac{1}{3 !} \frac{d^{3} \mathscr{V}}{d \phi_{0}{ }^{3}}\right|_{\phi_{0}=0}= & g_{B}-3 \lambda_{B}\left(\Omega_{0}{ }^{2 \prime}\right) I_{-1}\left(\Omega_{0}{ }^{2}\right)-\frac{3}{4} g_{B} \Omega_{0}{ }^{\prime \prime \prime} I_{-1}\left(\Omega_{0}{ }^{2}\right)+\frac{9}{8} g_{B}\left(\Omega_{0}{ }^{2 \prime}\right)^{2} I_{-2}\left(\Omega_{0}{ }^{2}\right) \\
& +\frac{1}{8}\left(\Omega_{0}{ }^{2 \prime}\right) \Omega_{0}{ }^{2 \prime \prime} I_{-1}\left(\Omega_{0}{ }^{2}\right)\left[1+6 \lambda_{B} I_{-1}\left(\Omega_{0}{ }^{2}\right)\right]-\frac{1}{8}\left(\Omega_{0}{ }^{2 \prime}\right)^{3} I_{-2}\left(\Omega_{0}{ }^{2}\right)\left[1+9 \lambda_{B} I_{-1}\left(\Omega_{0}{ }^{2}\right)\right],
\end{aligned}
$$

and the renormalized quartic one is

$$
\begin{aligned}
\lambda=\left.\frac{d^{4} \mathscr{V}}{d \phi_{0}^{4}}\right|_{\phi_{0}=0}= & \lambda_{B}-\frac{3}{2} \lambda_{B} \Omega_{0}^{2 \prime \prime} I_{-1}\left(\Omega_{0}{ }^{2}\right)+\frac{9}{4} \lambda_{B}\left(\Omega_{0}{ }^{2}\right) I_{-2}\left(\Omega_{0}{ }^{2}\right)+\frac{9}{8} g_{B}\left(\Omega_{0}{ }^{\prime \prime}\right) \Omega_{0}{ }^{2 \prime \prime} I_{-2}\left(\Omega_{0}{ }^{2}\right) \\
& -\frac{1}{4} g_{B} \Omega_{0}{ }^{2 \prime \prime \prime} I_{-1}\left(\Omega_{0}{ }^{2}\right)-\frac{15}{16} g_{B}\left(\Omega_{0}{ }^{\prime \prime}\right)^{3} I_{-3}\left(\Omega_{0}{ }^{2}\right) \\
& +\frac{1}{96}\left[4 \Omega_{0}{ }^{2 \prime \prime \prime}\left(\Omega_{0}{ }^{2 \prime}\right)+3\left(\Omega_{0}{ }^{\prime \prime \prime}\right)^{2}\right] I_{-1}\left(\Omega_{0}{ }^{2}\right)\left[1+6 \lambda_{B} I_{-1}\left(\Omega_{0}{ }^{2}\right)\right]-\frac{3}{16}\left(\Omega_{0}{ }^{2 \prime}\right)^{2} \Omega_{0}{ }^{2 \prime \prime} I_{-2}\left(\Omega_{0}{ }^{2}\right)\left[1+9 \lambda_{B} I_{-1}\left(\Omega_{0}{ }^{2}\right)\right] \\
& +\frac{15}{128}\left(\Omega_{0}{ }^{2 \prime}\right)^{4}\left\{I_{-3}\left(\Omega_{0}{ }^{2}\right)\left[1+8 \lambda_{B} I_{-1}\left(\Omega_{0}{ }^{2}\right)\right]+\frac{18}{5} \lambda_{B} I_{-2}{ }^{2}\left(\Omega_{0}{ }^{2}\right)\right\}
\end{aligned}
$$


where

$$
\left(\Omega_{0}^{2 \prime \prime}\right)=3 \lambda_{B} \frac{8+3 I_{-2}\left(\Omega_{0}^{2}\right)\left(\Omega_{0}^{2 \prime}\right)^{2}}{1+6 \lambda_{B} I_{-1}\left(\Omega_{0}^{2}\right)},
$$

which is again obtained by minimization of $\lambda$ or equivalently by minimization of $\mathscr{V}_{G}\left(\phi_{0}, \Omega\right)$ around $\phi_{0}=0$.

In all the above, stability requires that

$$
1+6 \lambda_{B} I_{-1}\left(\Omega_{0}^{2}\right)>0 \text {. }
$$

The following recurrence formula has been repeatedly used:

$$
\frac{d I_{n}\left(\Omega^{2}\right)}{d \Omega^{2}}=\left(n-\frac{1}{2}\right) I_{n-1}\left(\Omega^{2}\right) .
$$

We will also use the following expressions: ${ }^{5}$

$$
\begin{aligned}
& I_{1}\left(\Omega^{2}\right)-I_{1}\left(m^{2}\right)= \frac{1}{2}\left(\Omega^{2}-m^{2}\right) I_{0}\left(m^{2}\right) \\
&-\frac{1}{8}\left(\Omega^{2}-m^{2}\right)^{2} I_{-1}\left(m^{2}\right)+\Delta\left(\Omega^{2}, m^{2}\right), \\
& \Delta\left(\Omega^{2}, m^{2}\right)=\frac{1}{128 \pi^{2}}\left[\begin{array}{l}
2 \Omega^{4} \ln \frac{\Omega^{2}}{m^{2}}-2 m^{2}\left(\Omega^{2}-m^{2}\right) \\
\left.-3\left(\Omega^{2}-m^{2}\right)^{2}\right]+O\left(\frac{1}{\Lambda^{2}}\right),
\end{array}\right.
\end{aligned}
$$

$$
\begin{aligned}
& I_{0}\left(\Omega^{2}\right)-I_{0}\left(m^{2}\right)=-\frac{1}{2}\left(\Omega^{2}-m^{2}\right) I_{-1}\left(m^{2}\right)+\Gamma\left(\Omega^{2}, m^{2}\right), \\
& \Gamma\left(\Omega^{2}, m^{2}\right)=\frac{1}{16 \pi^{2}}\left(\Omega^{2} \ln \frac{\Omega^{2}}{m^{2}}-\Omega^{2}+m^{2}\right)+O\left(\frac{1}{\Lambda^{2}}\right) \\
& =2 \frac{d \Delta\left(\Omega^{2}, m^{2}\right)}{d \Omega^{2}}>0, \\
& I_{-1}\left(\Omega^{2}\right)=I_{-1}\left(m^{2}\right)=-\frac{1}{8 \pi^{2}} \ln \frac{\Omega^{2}}{m^{2}}+O\left(\frac{1}{\Lambda^{2}}\right) \\
& =-2 \frac{d \Gamma\left(\Omega^{2}, m^{2}\right)}{d \Omega^{2}}, \\
& I_{-1}\left(\Omega^{2}\right)=\frac{1}{8 \pi^{2}}\left[\ln \frac{4 \Lambda^{2}}{\Omega^{2}}-2\right)+O\left(\frac{1}{\Lambda^{2}}\right] .
\end{aligned}
$$

We now subtract an UV-divergent constant that contains the zero-point energy by considering

$$
\epsilon\left(\phi_{0}, \Omega\left(\phi_{0}\right)\right) \equiv \mathscr{V}_{G}\left(\phi_{0}, \Omega\left(\phi_{0}\right)\right)-\mathscr{V}_{G}\left(0, \Omega_{0}\right),
$$

which using Eq. (20) gives

$$
\begin{aligned}
\epsilon\left(\phi_{0}, \Omega\left(\phi_{0}\right)\right)= & \frac{1}{8}\left(\Omega^{2}-\Omega_{0}{ }^{2}\right)^{2} I_{-1}\left(\Omega_{0}{ }^{2}\right)+\Delta\left(\Omega^{2}, \Omega_{0}{ }^{2}\right)-\frac{1}{2}\left(\Omega^{2}-\Omega_{0}{ }^{2}\right) \Gamma\left(\Omega^{2}, \Omega_{0}{ }^{2}\right) \\
& +3 \lambda_{B}\left[\phi_{0}{ }^{2}-\frac{1}{2}\left(\Omega^{2}-\Omega_{0}{ }^{2}\right) I_{-1}\left(\Omega_{0}{ }^{2}\right)+\Gamma\left(\Omega^{2}, \Omega_{0}{ }^{2}\right)\right]^{2} \\
& +3 g_{B} \phi_{0}\left[-\frac{1}{2}\left(\Omega^{2}-\Omega_{0}{ }^{2}\right) I_{-1}\left(\Omega_{0}{ }^{2}\right)+\Gamma\left(\Omega^{2}, \Omega_{0}{ }^{2}\right)\right]+a \phi_{0}+\frac{1}{2} \Omega_{0}{ }^{2} \phi_{0}{ }^{2}+g_{B} \phi_{0}{ }^{3}-2 \lambda_{B} \phi_{0}{ }^{4}
\end{aligned}
$$

where $\Omega$ is $\Omega\left(\phi_{0}\right)$, the function of $\phi_{0}$ that minimizes the energy density. Of course this expression can also be written as

$\epsilon\left(\Omega_{0}, \Omega\left(\phi_{0}\right)\right)=a \phi_{0}+\frac{1}{2} m^{2} \phi_{0}{ }^{2}+g \phi_{0}{ }^{3}+\lambda \phi_{0}{ }^{4}+f\left(\phi_{0}\right)$,

where $f\left(\phi_{0}\right)$ is a function whose first four derivatives at $\phi_{0}=0$ are zero. Notice that for fixed $\phi_{0}$ and large $\Omega$, $\epsilon\left(\phi_{0}, \Omega\right)$ gives, keeping dominant and subdominant terms,

$$
\begin{aligned}
& \epsilon\left(\phi_{0}, \Omega\right) \underset{\Omega \underset{\text { large }}{\approx}}{\approx} \frac{1}{8} \Omega^{4} I_{-1}\left(\Omega_{0}{ }^{2}\right)\left[1+6 \lambda_{B} I_{-1}\left(\Omega_{0}{ }^{2}\right)\right] \\
&-\frac{1}{64 \pi^{2}} \Omega^{4} \ln \frac{\Omega^{2}}{\Omega_{0}{ }^{2}}\left[1+12 \lambda_{B} I_{-1}\left(\Omega_{0}{ }^{2}\right)\right],
\end{aligned}
$$

which from Eq. (18) can easily be seen to be positive. Thus $\Omega\left(\phi_{0}\right)$ is either given by

$$
\Omega=0
$$

or by a solution of

$$
\begin{aligned}
6 \lambda_{B}\left[2 \phi_{0}{ }^{2}-\left(\bar{\Omega}^{2}-\Omega_{0}{ }^{2}\right) I_{-1}\left(\Omega_{0}{ }^{2}\right)+2 \Gamma\left(\bar{\Omega}^{2}, \Omega_{0}{ }^{2}\right)\right]+6 g_{B} \phi_{0} \\
=\bar{\Omega}^{2}-\Omega_{0}{ }^{2} .
\end{aligned}
$$

One can easily check that Eqs. (13) and (17) can be obtained by differentiating Eq. (26) as expected.

\section{A STABLE PRECARIOUS THEORY}

We now consider all possible values of $\lambda_{B}$ compatible with Eq. (18). The smallest value is

$$
\begin{gathered}
\lambda_{B}=-\frac{1}{6 I_{-1}\left(\Omega_{0}^{2}\right)}+\frac{C_{1}}{I_{-1}{ }^{2}\left(\Omega_{0}{ }^{2}\right)}+O\left[\frac{1}{I_{-1}{ }^{3}\left(\Omega_{0}{ }^{2}\right)}\right], \\
C_{1}>0 .
\end{gathered}
$$

The finiteness of $g$ implies from Eqs. (13) and (15) that

$$
g_{B}=\frac{C_{2}}{I_{-1}\left(\Omega_{0}{ }^{2}\right)}+O\left(\frac{1}{I_{-1}{ }^{2}\left(\Omega_{0}{ }^{2}\right)}\right) \text {. }
$$

Notice that Eqs. (27) and (28) imply that $\epsilon\left(\phi_{0}, \Omega\left(\phi_{0}\right)\right)$ given by Eq. (22) is finite when $\Lambda \rightarrow \infty$ : the theory is correctly renormalized. Equations (13) and (17) give renormalized mass and coupling constants that are functions of $\Omega_{0}, C_{1}$, and $C_{2}$ :

$$
\begin{aligned}
& m^{2}=\Omega_{0}^{2}-\frac{3}{2} \frac{C_{2}^{2}}{C_{1}}, \\
& g=\frac{C_{2}}{2 C_{1}}+\frac{1}{192 \pi^{2} \Omega_{0}^{2}}\left(\frac{C_{2}}{C_{1}}\right]^{3}, \\
& \lambda=-\frac{1}{768 C_{1}}\left[64+4\left[\frac{C_{2}}{\pi \Omega_{0} C_{1}}\right]^{2}\right. \\
& \left.\quad+\left(C_{1} \pi^{2}+\frac{1}{16}\right)\left[\frac{C_{2}}{\pi \Omega_{0} C_{1}}\right)^{4}\right] .
\end{aligned}
$$

The theory is interacting. Equation (26) now reads 


$$
\phi_{0}{ }^{2}+\Gamma\left(\bar{\Omega}^{2}, \Omega_{0}{ }^{2}\right)-3 C_{2} \phi_{0}+3 C_{1}\left(\bar{\Omega}^{2}-\Omega_{0}{ }^{2}\right)=0 .
$$

Obviously, this equation does not have solutions if $\phi_{0}$ is beyond some critical value (recall $\Gamma \geq 0$ and $C_{1}>0$ ). Then necessarily the $\Omega=0$ solution will be operative. Equation (22) now reads for this value of $\Omega$

$\epsilon\left(\phi_{0}, 0\right)=\left[\frac{3}{4} C_{1}-\frac{1}{128 \pi^{2}}\right] \Omega_{0}{ }^{4}+\left(a+\frac{3}{2} C_{2} \Omega_{0}{ }^{2}\right) \phi_{0}$,

which is only bounded from below if

$$
a+\frac{3}{2} C_{2} \Omega_{0}^{2}=0 \text {. }
$$

Even then the theory is only precarious, because there is a contribution to Eq. (31) that for finite $\Lambda$ is dominant for large $\phi_{0}$ :

$$
\epsilon\left(\phi_{0}, 0\right) \underset{\phi_{0} \text { 吕ge }}{\approx}-\frac{\phi_{0}{ }^{4}}{6 I_{-1}\left(\Omega_{0}{ }^{2}\right)}
$$

leading to instability. Thus, in some regions of the parameter space of $\Omega_{0}, C_{1}$, and $C_{2}$ one has a ground state separated from the unbounded well by a barrier that becomes of infinite width when $\Lambda \rightarrow \infty$.

Equation (32) is, in fact, hiding a very interesting result. This condition in terms of the renormalized parameters implies that the original bare potential must be symmetric for the theory to be stable. One can see this by asking under which conditions is the original potential of Eq. (1) symmetric about some $\phi_{0}$. In terms of the bare parameters this condition is

$$
a_{B}-\frac{m_{B}^{2} g_{B}}{4 \lambda_{B}}+\frac{1}{8} \frac{g_{B}^{3}}{\lambda_{B}^{2}}=0 \text {. }
$$

With the use of Eqs. (11), (27), and (28) and dropping terms of $O\left(1 / I_{-1}\right)$, this becomes Eq. (32). Thus the requirement of stability on the most general asymmetric $\phi^{4}$ theory reduces the theory to its symmetric version. ${ }^{12}$

\section{OTHER POSSIBILITIES?}

Consider now a theory with

$$
\lambda_{B}=\frac{C_{1}}{6 I_{-1}\left(\Omega_{0}{ }^{2}\right)}+O\left(\frac{1}{I_{-1}{ }^{2}\left(\Omega_{0}{ }^{2}\right)}\right), \quad C_{1}>-1 .
$$

An interacting theory requires in this case

$$
g_{B}=C_{2}+O\left(\frac{1}{I_{-1}\left(\Omega_{0}^{2}\right)}\right) \text {. }
$$

Then Eq. (14) implies

$$
m^{2}=\Omega_{0}^{2}-9 \frac{C_{2}^{2}}{1+C_{1}} I_{-1}\left(\Omega_{0}^{2}\right)+\text { finite terms } .
$$

The finiteness of the renormalized mass demands that $\Omega_{0}{ }^{2}$ grows with $\Lambda$ as $I_{-1}\left(\Omega_{0}{ }^{2}\right)$. One can easily see that this implies $\lambda=0$, but $g \neq 0$. Equation (26) now reads

$$
6 C_{2} \phi_{0}=\left(1+C_{1}\right)\left(\bar{\Omega}^{2}-\Omega_{0}{ }^{2}\right),
$$

which implies the energy density as given in Eq. (22) is not finite when $\Lambda \rightarrow \infty$. The theory is not correctly renormalized.

Consider finally

$$
\lambda_{B}=C_{1}+O\left(\frac{1}{I_{-1}\left(\Omega_{0}^{2}\right)}\right), \quad C_{1}>0
$$

Finiteness of the renormalized coupling constants demands

$$
g_{B}=C_{2}+O\left(\frac{1}{I_{-1}\left(\Omega_{0}^{2}\right)}\right)
$$

Then

$$
\begin{aligned}
& m^{2}=\Omega_{0}^{2}-\frac{3}{2} \frac{C_{2}^{2}}{C_{1}}, \\
& g=-2 C_{2}, \\
& \lambda=-2 C_{1},
\end{aligned}
$$

and Eq. (26) reads

$$
C_{1}\left[2 \phi_{0}{ }^{2}-\left(\Omega^{2}-\Omega_{0}^{2}\right) I_{-1}\left(\Omega_{0}^{2}\right)\right]+C_{2} \phi_{0}=0,
$$

which always has a solution. Substituting into Eq. (22) one finds

$$
\epsilon\left(\phi_{0}\right)=a \phi_{0}+\frac{1}{2} m^{2} \phi_{0}^{2}+g \phi_{0}^{3}+\lambda \phi_{0}^{4},
$$

which is certainly finite when $\Lambda \rightarrow \infty$, but unbound from below.

We return briefly to the case when Eq. (10) is valid. Stability requires that

$$
m_{B}^{2}+12 \lambda_{B} I_{0}(0)<0 \text {. }
$$

It is easy to convince oneself that also all the derivatives of $\Omega^{2}$ at $\phi_{0}=0$ are zero. This gives

$$
\begin{aligned}
& a=a_{B}+3 g_{B} I_{0}(0), \\
& m^{2}=m_{B}^{2}+12 \lambda_{B} I_{0}(0), \\
& g=g_{B}, \\
& \lambda=\lambda_{B} .
\end{aligned}
$$

The subtracted energy density is

$$
\begin{aligned}
\epsilon\left(\phi_{0}, \Omega\left(\phi_{0}\right)\right)= & -\frac{1}{128 \pi^{2}} \Omega^{4}+\frac{1}{4}\left(2 m^{2}-\Omega^{2}\right)\left[-\frac{1}{16 \pi^{2}} \Omega^{2}\left[\ln \frac{4 \Lambda^{2}}{\Omega^{2}}-1\right]\right]+3 \lambda_{B}\left[\phi_{0}^{2}-\frac{1}{16 \pi^{2}} \Omega^{2}\left[\ln \frac{4 \Lambda^{2}}{\Omega^{2}}-1\right]\right]^{2} \\
& +3 g_{B} \phi_{0}\left[-\frac{1}{16 \pi^{2}} \Omega^{2}\left[\ln \frac{4 \Lambda^{2}}{\Omega^{2}}-1\right]\right]+a \phi_{0}+\frac{1}{2} m^{2} \phi_{0}{ }^{2}+g_{B} \phi_{0}{ }^{3}-2 \lambda_{B} \phi_{0}^{4}
\end{aligned}
$$


Now $\Omega\left(\phi_{0}\right)$ is given either by

$$
\Omega=0
$$

or by a solution of

$6 \lambda_{B}\left[2 \phi_{0}{ }^{2}-\frac{1}{8 \pi^{2}} \bar{\Omega}^{2}\left[\ln \frac{4 \Lambda^{2}}{\Omega^{2}}-1\right]\right]+6 g_{B} \phi_{0}=\bar{\Omega}^{2}-m^{2}$.

In the first case one finds from Eq. (46)

$$
\epsilon\left(\phi_{0}, 0\right)=a \phi_{0}+\frac{1}{2} m^{2} \phi_{0}{ }^{2}+g \phi_{0}{ }^{3}+\lambda \phi_{0}{ }^{4},
$$

which requires $\lambda>0$. However, for large $\phi_{0}$ when solutions to Eq. (48) exist, one has

$$
\epsilon\left(\phi_{0}, \bar{\Omega}\right) \underset{\text { large } \phi_{0}}{\approx}-2 \lambda \phi_{0}^{4},
$$

which is unbounded.

\section{CONCLUSION}

We have studied the existence of a stable ground state in the most general, renormalizable, single scalar field theory within the variational Gaussian approximation. Our results generalize Stevenson's ${ }^{5}$ for even $\phi^{4}$ theory. Indeed in certain regions of parameter space an interacting finite theory exists and is stable once the cutoff is sent to infinity.

Perhaps the most startling result of our analysis is that the only viable $\phi^{4}$ theory is one that is symmetric.

\section{ACKNOWLEDGMENTS}

The authors gratefully acknowledge conversations with P. M. Stevenson. One of us (R.K.) acknowledges NSERC Canada for financial support. The other (R.T.) thanks Roman Koniuk for his warm hospitality and acknowledges financial support by CAICYT Spain, Contract No. 0435 .
*Permanent address: Department of Theoretical Physics, University of Barcelona, Diagonal 647, Barcelona 28, Spain.

${ }^{1}$ K. G. Wilson, Phys. Rev. D 6, 419 (1972) (Ref. 8).

${ }^{2}$ M. Aizenman, Phys. Rev. Lett. 47, 1 (1981); Commun. Math. Phys. 86, 1 (1982).

${ }^{3}$ J. Fröhlich, Nucl. Phys. B200, 281 (1982); C. Aragao de Carvalho, S. Caracciolo, and J. Fröhlich, ibid. B215, 209 (1983).

${ }^{4}$ D. J. E. Callaway and R. Petronzio, Nucl. Phys. B240, 577 (1984).
${ }^{5}$ P. M. Stevenson, Z. Phys. 24, 87 (1984).

${ }^{6}$ L. I. Schiff, Phys. Rev. 130, 458 (1963).

${ }^{7}$ T. Barnes and G. I. Ghandour, Phys. Rev. D 22, 924 (1980).

${ }^{8}$ S. Coleman and E. Weinberg, Phys. Rev. D 7, 1888 (1973).

${ }^{9}$ W. A. Bardeen and M. Moshe, Phys. Rev. D 28, 1372 (1983).

${ }^{10}$ W. A. Bardeen, M. Moshe, and M. Bander, Phys. Rev. Lett. 52, 1188 (1984).

${ }^{11}$ P. M. Stevenson, Phys. Rev. D 30, 1712 (1984).

${ }^{12}$ We thank P. M. Stevenson for providing this insight. 